\title{
Nivel de conocimiento sobre prevención de principales enfermedades bucodentales en estudiantes universitarios Cuenca-Ecuador
}

\section{Level of knowledge about prevention of major oral diseases in university students Cuenca-Ecuador}

\author{
Zulay Bastidas Calva ${ }^{1 *}$, Lissette Alejandra Cerón Miranda ${ }^{1}$ y Liliana Encalada Verdugo ${ }^{1}$ \\ ${ }^{1}$ Universidad Católica de Cuenca \\ *mbastidasc@ucacue.edu.ec
}

DOI: https://doi.org/10.26871/killkana_salud.v2i2.332

\begin{abstract}
Resumen
Objetivo: el objetivo de esta investigación fue determinar el nivel de conocimiento sobre prevención de las principales enfermedades bucodentales en los estudiantes de la Universidad Católica de Cuenca año 2017. Materiales y Metodos: Estudio descriptivo de corte transversal, donde participaron 364 estudiantes, que completaron una encuesta con 21 preguntas divididas en tres bloques sobre salud bucal, enfermedades bucodentales y prevención de salud bucal. Resultados: Pertenecían al sexo femenino $51 \%$ y sexo masculino $49 \%$, el promedio de edad fue entre 19 y 24 años. El nivel de conocimiento fue medido en escalas correspondiendo a bajo el $1 \%$, media baja el $28 \%$, media alta el $58 \%$ y alta con un $13 \%$. La facultad de medicina fue la que registro mayor nivel de conocimiento con un $86 \%$ en la escala de alta. El $59 \%$ de los estudiantes poseen un nivel alto de conocimiento de las principales enfermedades bucodentales y el $76 \%$ conoce acerca de prevención de salud bucal. Conclusiones: Los estudiantes de la Universidad Católica de Cuenca Sede Matriz tienen un nivel de conocimiento aceptable ya que los mayores porcentajes se registraron en las escalas de media baja y media alta siendo mayor el nivel de conocimiento en la facultad de Medicina.
\end{abstract}

Palabras clave: conocimiento, salud, caries, gingivitis, maloclusión.

\begin{abstract}
Objective: The objective of this research was to determine the level of knowledge on prevention of the main oral diseases in students of the Catholic University of Cuenca year 2017. Materials and methods: A descriptive cross-sectional study, which was attended by 364 students, who completed a survey with 21 questions divided into three blocks on oral health, oral diseases and prevention of oral health. Results: belonged to the $51 \%$ female and $49 \%$ male, the average age was between 19 and 24 years. The level of knowledge was measured on a scale corresponding to under 1\%, average 28\% lower, 58\% high average and high with a 13\%. The faculty of medicine was the highest level of knowledge with a 86 per cent in the scale of high. The 59\% of the students have a high level of knowledge of the main oral diseases and 76 per cent know about prevention of oral health. Conclusions: It was concluded that the students of the Catholic University of Cuenca Headquarters have an acceptable level of knowledge as the highest percentages were recorded in the scales of lower middle and upper middle being higher in the faculty of medicine.
\end{abstract}

Key words: knowledge, health, dental caries, gingivitis, malocclusion.

\section{Introducción}

Se considera a la salud bucal como parte fundamental de la salud general, ya que si una persona presenta alguna enfermedad bucal no puede ser considerado como completamente saludable. La falta de conocimiento en cuanto a salud oral es uno de los problemas más graves en la sociedad ya que debido a esto se va incrementando la aparición de enfermedades bucodentales. Esta investigación pretende determinar el nivel de conocimiento que tienen los estudiantes de la Universidad Católica de Cuenca acerca de la prevención de enfermedades bucodentales con la finalidad de incrementar e implementar en el futuro programas de prevención en esta población. La caries dental, las enfermedades periodontales y las maloclusiones a pesar de ser bastante prevalentes a nivel mundial son enfermedades a las que se les ha dado menor importancia por no ser causantes de mortalidad directa, pero su alta frecuencia en 
la población hace que se convierta en un problema de salud pública y por ello es necesaria una atención integral. ${ }^{1}$

La salud oral se relaciona directamente con la calidad de vida de las personas puesto que la boca es responsable de actividades fundamentales como son el lenguaje, expresiones faciales, masticación, deglución, salivación, sentido del gusto; etc. ${ }^{2}$ Dentro de la salud oral debe considerarse como parte fundamental la educación ya que a través de ella se puede implementar programas preventivos para la sociedad. Los profesionales de la salud deben educar a sus pacientes a través de la motivación, para así lograr cambios constantes en sus hábitos, obteniendo resultados positivos que logren mejorar su salud bucal y reducir el desarrollo de las enfermedades bucodentales. ${ }^{3}$

Además la salud bucal ejerce un gran impacto social ya que al estar relacionada con el dolor ocasiona dificultades en cuanto a la alimentación, comunicación así como también la satisfacción estética, siendo factores muy importantes para el desarrollo físico y mental de todas las personas. ${ }^{4}$ Por ello un mayor conocimiento de salud bucal pueden llevar a mejorar acciones en cuanto a la prevención y control de las enfermedades bucales en sus etapas iniciales así como también aumentar el uso de servicios de salud odontológica. ${ }^{5}$

Según la Organización Mundial de la Salud (OMS) se define a la salud bucodental como la ausencia de enfermedades y trastornos que afectan a dientes, mucosas, músculos, huesos y todas aquellas estructuras que conforman la cavidad bucal y que están directamente relacionadas con actividades como la masticación, comunicación oral y función musculo facial, es decir un estado de normalidad y funcionalidad de todas estas estructuras. ${ }^{6}$

La caries dental es una enfermedad crónica, infecciosa, multifactorial y transmisible de los dientes, caracterizada por la destrucción progresiva de los tejidos calcificados debido a la acción de microorganismos. ${ }^{7}$ Este proceso ocurre en la estructura dentaria que está en contacto con los depósitos de microorganismos y que debido al desequilibrio entre la sustancia dental y el fluido de la placa circundante, trae como resultado una pérdida del mineral de la superficie dental, cuyo signo clínico es una destrucción localizada de tejidos duros. ${ }^{8}$

Las enfermedades periodontales se producen como consecuencia de la interacción entre la acción de bacterias y la respuesta del huésped, produciendo daños a nivel de las estructuras de soporte y protección del diente como son la encía, ligamento periodontal, cemento y hueso alveolar. Intervienen también factores ambientales y genéticos que contribuyen al desarrollo de la enfermedad ya que vuelven más susceptibles a los individuos, entre estos tenemos el consumo de alcohol, el tabaco, la mala alimentación, una higiene oral deficiente, el estrés, mal posiciones dentarias; etc. ${ }^{9}$ La enfermedad periodontal tiene una relación directa con el biofilm, que es el depósito de microorganismos que se encuentran adheridos a la superficie dental. ${ }^{10}$
Entre estas enfermedades están la gingivitis que es un proceso inflamatorio de la encía, considerado como reversible si se aplica un tratamiento a tiempo, se pueden presentar cambios de color, edema y sangrado en las encías, la acumulación de placa bacteriana constituye el principal factor que determina su aparición. ${ }^{11} \mathrm{Y}$ la periodontitis que provoca la destrucción de las estructuras de soporte de los dientes como son el ligamento periodontal, hueso alveolar y cemento radicular, su evolución es lenta pero continua y su severidad depende de la cantidad de placa y cálculo dental presente en los dientes. ${ }^{12}$ Entre los signos que la caracterizan están el sangrado al sondaje, halitosis, bolsas periodontales mayores a $4 \mathrm{~mm}$, pérdida de la inserción epitelial y ósea, movilidad dental y migraciones patológicas. ${ }^{13}$

La maloclusión es definida como la oclusión anormal en la que las piezas dentales no se encuentran en una posición correcta de manera que logren un contacto adecuado con las piezas dentales antagonistas, son variaciones de crecimiento y morfología. En cuanto a su etiología esta puede estar ligada a factores genéticos, ambientales, una combinación de los dos y en otros casos puede ser el resultado de hábitos orales. ${ }^{14}$ Según la OMS, las maloclusiones corresponden a la tercera enfermedad bucal más frecuente, después de la caries y la enfermedad periodontal. Conocer acerca de este problema es primordial para aplicar programas preventivos, interceptivos y de tratamiento. ${ }^{15}$

Las enfermedades bucodentales forman parte de los problemas de salud pública por su prevalencia e impacto sobre los individuos ya que ocasionan dolor, malestar, limitación y discapacidad social y funcional. Un estilo de vida incorrecto sumado a los hábitos insanos adquiridos durante nuestra vida constituyen los principales factores de riesgo asociados a las enfermedades bucodentales, siendo entonces muy importante la educación para la salud, la misma que fue definida por la OMS como un campo especial de las Ciencias de la Salud que tiene como objetivo trasmitir los conocimientos, así como fomentar conductas que favorezcan la conservación de la salud de las personas. Siendo así la educación el instrumento más eficaz para lograr un cambio de actitud que nos conduzca hacia una vida más saludable. ${ }^{16}$

La prevención se define como "cualquier medida que permita reducir la posibilidad de aparición de una afectación o enfermedad, o bien interrumpir o aminorar su progresión". Entonces no se trata solo de evitar la aparición de una enfermedad o afectación, sino también de detener su progreso para lograr así la cura de la misma y si esto no es posible tratar de reducir su progresión al máximo de tiempo. $^{17}$

Dentro de la prevención está la higiene oral que se define como el conjunto de medidas físicas y químicas encaminadas al control de la formación de placa bacteriana que es el principal factor para el desarrollo de caries y enfermedad periodontal, así como también la limpieza de la lengua, mantenimiento de tejidos y estructuras dentales. ${ }^{18}$ Los hábitos que se practican a diario juegan un papel muy 
importante para mantener una buena salud oral, el tabaquismo, el consumo excesivo de azucares, bebidas dulces, etc, son los que atacan a nuestra dentición y la vuelven susceptible a la caries. ${ }^{19}$

Para lograr una higiene oral adecuada es fundamental el cepillado dental que nos permite remover la placa bacteriana de la superficie de los dientes y encías, es recomendable que nos cepillemos los dientes tres veces al día, especialmente después de las comidas. ${ }^{20}$ El cepillado se vuelve más eficaz con el uso de un dentífrico adecuado, sobre todo los que poseen flúor ya que ayudan en la prevención de caries dental. ${ }^{21}$ Esto debe complementarse con el uso de seda o hilo dental ya que en la mayoría de los casos las fibras de nylon del cepillo no llegan a los espacios interproximales para su completa limpieza, siendo estos sitios en donde por lo general se inician los procesos cariosos. ${ }^{22}$

\section{Metodología}

El estudio se aplicó a los 6617 estudiantes matriculados en la Universidad Católica de Cuenca durante el periodo 2016-2017, que tengan entre 18 y 24 años de edad, excepto los estudiantes pertenecientes a la facultad de Odontología. A través de la fórmula de muestreo la encuesta se aplicaría a 364 estudiantes.

Fórmula de muestreo:

$$
n=\frac{Z^{2} * p * q * N}{Z^{2} * p * q+E^{2}(N-1)}
$$

Dónde:

$Z^{2}=$ nivel de confianza (1.96)

$\mathbf{p}=$ probabilidad de selección $(0.5)$

$\mathbf{q}=$ probabilidad de fracaso $(0.5)$

$\mathbf{N}=$ tamaño de la población (6617)

$E^{2}=$ error permitido $(0.0025)$

El examinador empezó por solicitar a través de un oficio dirigido al rector de la Universidad Católica de Cuenca se le facilite el número de estudiantes de cada facultad, siendo esto necesario para determinar la muestra, una vez que se obtuvo una respuesta favorable por parte del departamento de informática, quienes fueron los que dieron a conocer el número de estudiantes de cada facultad, posteriormente se procedió a elaborar un banco de preguntas acerca de salud bucal, las tres principales enfermedades bucodentales y la prevención de las mismas, una vez que ya se elaboró la encuesta se envió a 8 expertos a quienes se les indicó los objetivos de la investigación y los parámetros que debían ser calificados en cada uno de los ítems que fueron pertinencia, relevancia y claridad para así obtener la validación de dicha encuesta, ya con los resultados de cada uno de los expertos se ingresaron las calificaciones de cada ítem a una base de datos y aplicando el coeficiente $\mathrm{V}$ de Aiken se obtuvo la validación de la encuesta por parte de expertos con un resultado final de 0.89 , ya con estos resultados se aplicó a los estudiantes de cada facultad de la Universidad Católica de Cuenca Sede Matriz.
La encuesta validada por expertos constó de 3 partes, cada una con 7 preguntas, la primera correspondía a salud bucal, la segunda a enfermedades bucodentales y la tercera a prevención.

La encuesta fue aplicada en forma aleatoria a los estudiantes mediante un proceso de aleatoriedad del total de la población.

Para el registro de los datos, se tomó en cuenta las encuestas aplicadas a los estudiantes de la Universidad Católica de Cuenca Sede Matriz, las cuales fueron ingresadas a una base de datos en el programa EXCEL, estas encuestas reflejan el nivel de conocimiento sobre la prevención de las principales enfermedades bucodentales que fue registrado con las siguientes características:

Finalizada la aplicación de las encuestas se procedió a enumerar cada una, para así después en una base de datos ingresar lo siguiente:

1) La edad y sexo de los estudiantes encuestados.

2) La facultad a la que pertenecen cada estudiante encuestado.

3) Cada uno de los ítems con su respectiva calificación.

Cuando se realiza la calificación de cada uno de los ítems se le asigna el valor de 1 si la respuesta fue respondida correctamente; 0.5 si se seleccionó una de las opciones para aquellas preguntas con más de una respuesta correcta y 0 si la respuesta fue incorrecta.

Con los datos obtenidos se procedió a desarrollar una tabla de datos en Microsoft Excel 2010, en donde se ingresó los datos como sexo, edad, facultad de los estudiantes encuestados así como también cada una de las preguntas de la encuesta, esta información fue organizada y al aplicar una serie de fórmulas se obtuvo los porcentajes de la distribución correspondiente al sexo de los estudiantes, a la facultad a la que pertenecen, así como también los porcentajes que reflejan el nivel de conocimiento en general de todos los estudiantes encuestados, el nivel de conocimiento por cada facultad, teniendo escalas entre baja, media baja, media alta $\mathrm{y}$ alta.

El presente estudio no implicó conflictos bioéticos, debido a que se ejecuta a través de la aplicación de una encuesta a los estudiantes de la Universidad Católica de Cuenca.

\section{Resultados}

Se evaluaron 364 estudiantes de entre 18 y 24 años de la Universidad Católica de Cuenca, siendo $49 \%$ de sexo masculino y $51 \%$ de sexo femenino. 


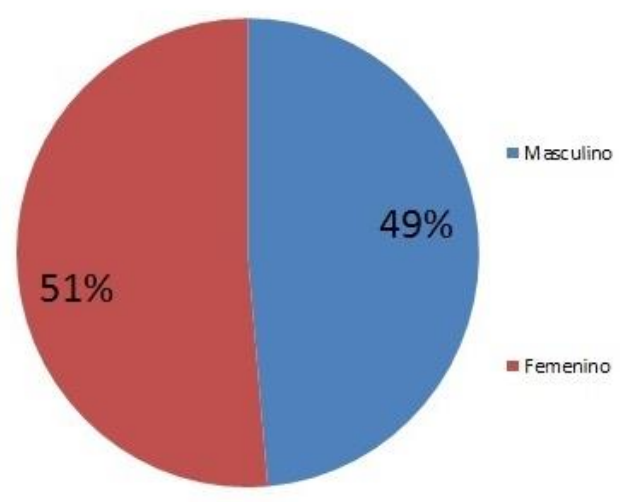

Fig. 1. Distribución de la muestra de acuerdo al sexo

El nivel de conocimiento fue registrado en cuatro escalas en donde 24 de los estudiantes encuestados alcanzaron la escala baja de conocimiento, 180 la escala media baja, seguido de la escala media alta con 135 estudiantes y la escala alta la alcanzaron 25 estudiantes.

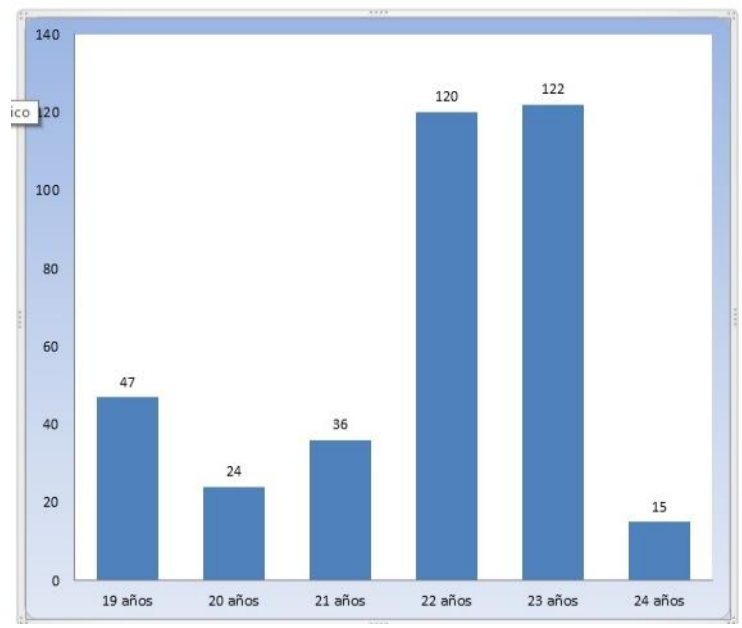

Fig. 2. Nivel de conocimiento

Además, los niveles de conocimiento de acuerdo a la carrera: corresponden a la facultad de inglés quien registró el más bajo nivel de conocimiento a diferencia de la facultad de Medicina que obtuvo el más alto nivel de conocimiento y las facultades restantes están entre la escala media alta y media baja.

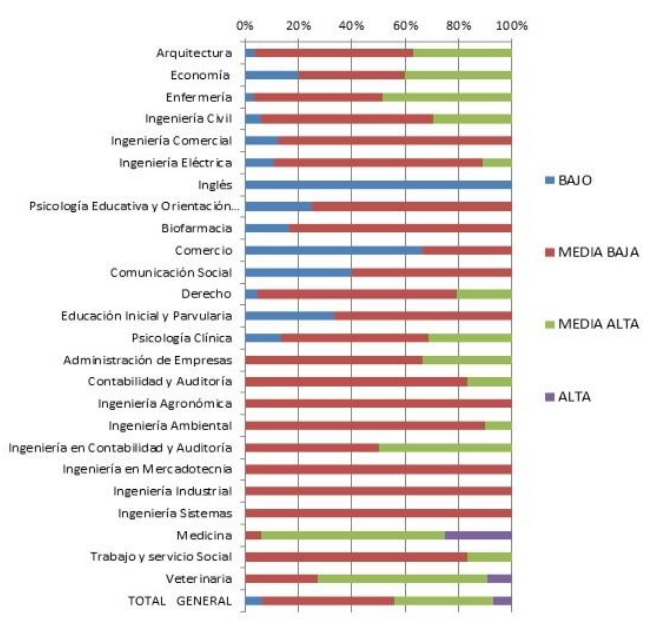

Fig. 3. Nivel de conocimiento correspondiente a cada facultad

El $59 \%$ de los estudiantes poseen un nivel alto de conocimiento de las principales enfermedades bucodentales y un porcentaje del $41 \%$ de los estudiantes tienen bajo nivel de conocimiento.

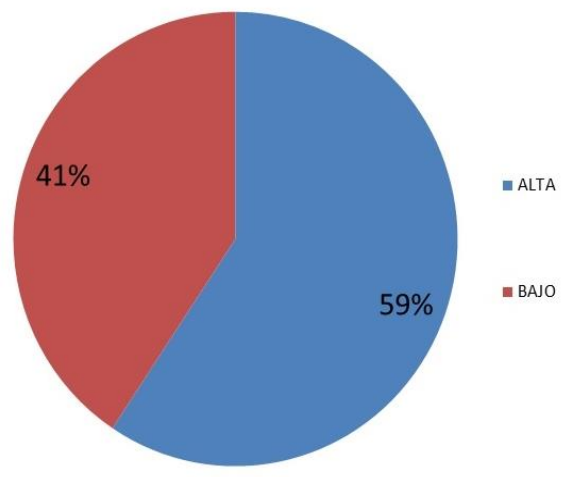

Fig. 4. Nivel de conocimiento de las principales enfermedades bucodentales

El $76 \%$ de los estudiantes poseen un nivel de conocimiento alto acerca de prevención en salud bucal y un $24 \%$ de estudiantes tienen un nivel de conocimiento bajo.

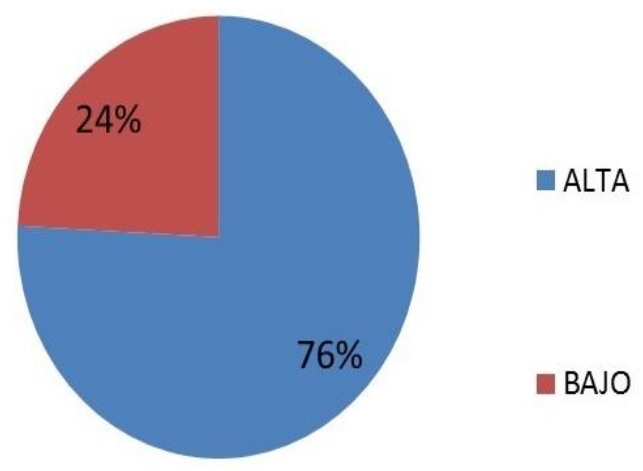

Fig. 5. Nivel de conocimiento sobre prevención de salud bucal 


\section{Discusión}

Al realizar esta investigación se determinó el nivel de conocimiento sobre prevención de las principales enfermedades enfermedades bucodentales por parte de los estudiantes de la Universidad Católica de Cuenca, el mismo que fue bajo, en donde 24 de los estudiantes encuestados alcanzaron esta escala correspondiendo al $7 \%$, la escala media baja la obtuvieron 180 estudiantes correspondiendo al $49 \%$, la media alta la obtuvieron 135 estudiantes que corresponde al $37 \%$ y alta la alcanzaron 25 estudiantes correspondiente a un $7 \%$ resultados que se asemejan al igual que en el estudio realizado por estudiantes de la facultad de Odontología de la Universidad de Cuenca en donde el nivel de conocimiento que se registró fue insuficiente en $8,4 \%$; aceptable en $43,5 \%$; buena en $41,2 \%$; muy buena en $6,9 \%{ }^{23}$ y el estudio realizado en la ciudad de Mysore a estudiantes preuniversitarios determino que aproximadamente el $67 \%$ de los estudiantes tiene conocimiento aceptable acerca de salud bucal, el $24 \%$ tienen bajo nivel y el $9 \%$ presentan un nivel de conocimiento alto. ${ }^{24}$

En cuanto a cada una de las facultades se determinó que la facultad de Medicina fue la que mostró mayor nivel de conocimiento sobre prevención de las principales enfermedades bucodentales, al igual que en el estudio realizado en la Universidad de Complutense de Madrid en el 2005 en donde los estudiantes de la facultad de Medicina también presentaron un alto nivel de conocimiento de enfermedades bucodentales. $^{25}$

Sobre el nivel de conocimiento de las principales enfermedades bucodentales se obtuvo resultados de $59 \%$ que tienen conocimiento y $41 \%$ desconocen acerca del tema, en comparación con el estudio realizado por Min-Kyoung Park, Hye-Jung Jin, Min-Kyung Lee en Korea el mismo que registró el nivel de conocimiento de las principales enfermedades bucodentales que fue del $73,5 \%$ correspondiente al nivel de conocimiento alto y el $23,5 \%$ a un nivel de conocimiento bajo. ${ }^{26}$

El nivel de conocimiento en cuanto a las medidas de prevención en los estudiantes fue de $76 \%$ en la escala de alto conocimiento y $24 \%$ que desconocen, similar al estudio de Díaz-Reissner, Pérez-Bejarano, SanabriaVázquez, Ferreira-GaonaI, Cueto-González, Barreto, ArceMena, Ledesma-Amarilla quienes llegaron a determinar que un elevado porcentaje de estudiantes tiene conocimiento acerca de las medidas de prevención bucal. ${ }^{23}$

Con la aplicación de la encuesta realizada para esta investigación se obtuvo como resultados que el nivel de conocimiento sobre prevención de las principales enfermedades bucodentales de los estudiantes de la Universidad Católica de Cuenca es bajo en un $7 \%$ siendo casi nulos sus conocimientos acerca de salud bucal, el $49 \%$ tiene un nivel de conocimiento medio bajo es decir que poseen poco conocimiento, el $37 \%$ posee un nivel medio alto con lo que demuestra que tienen conocimientos aceptables acerca de salud bucal mientras que solo el $7 \%$ alcanzo el nivel alto de conocimiento lo que demuestra que tan solo este míni- mo porcentaje de estudiantes tienen varios conocimientos acerca de salud bucal, cifras que encaminan a fomentar programas que incrementen el nivel de conocimiento sobre prevención ya que la salud bucal se relaciona directamente con la salud general de una persona.

Con respecto a nivel de conocimiento de los estudiantes de acuerdo a cada facultad fue Medicina la que registro el mayor nivel de conocimiento en la escala alta lo que hace pensar que existe un mayor porcentaje en esta facultad por ser una escuela directamente relacionada con la salud de la persona.

El nivel de conocimiento sobre las principales enfermedades bucodentales registró un porcentaje del $59 \%$ de los estudiantes que poseen un nivel alto de conocimiento y un porcentaje del $41 \%$ de los estudiantes que tienen bajo nivel de conocimiento, estos porcentajes no muy diferenciados indican que es necesario incentivar a los estudiantes universitarios a cambiar la actitud sobre la salud bucodental y el papel que juega está dentro de la salud en general.

El nivel de conocimiento sobre medidas de prevención en salud bucal es del $76 \%$ de los estudiantes que poseen un nivel de conocimiento alto acerca de prevención en salud bucal y un $24 \%$ de estudiantes que tienen un nivel de conocimiento bajo, lo que demuestra que es necesario, al ser bastante marcada la diferencia entre estos porcentajes lo importante de reforzar los conocimientos de los estudiantes que registran mayor porcentaje para lograr implementar nuevos programas preventivos para aquellos estudiantes que registran un nivel bajo de conocimiento.

\section{Referencias Bibliográficas}

1. Rodríguez Chala H, Solar Carballo O, Pazos Rodríguez A, Marrero Hernández M. Enfermedades bucales en estudiantes chinos. Revista Cubana de Estomatología. 2013;

2. Portal Médicos. Conocimiento de estudiantes de Enfermería sobre salud bucal. 2018 Dec;Available from: https://www.revista-portalesmedicos.com/revista-medica/ conocimiento-estudiantes-de-enfermeria-salud-bucal.

3. Gallardo-Schultz JC, Ávalos Márquez N, Huillca-Castillo Mn, Picasso-Pozo E, Omori-Mitumor A. NIVEL DE CONOCIMIENTOS EN SALUD ORAL RELACIONADO. KIRU. 2015;.

4. Cisneros Estala M, Tijerina González L, Cantú Martínez P. SALUD BUCAL Y HÁBITOS ALIMENTARIOS EN ESTUDIANTES UNIVERSITARIOS. RESPYN Revista de Salud Pública y Nutrición. 2010;11(4). Available from: http://respyn.uanl.mx/index.php/respyn/article/view/276.

5. Dho MS. Conocimientos de salud bucodental en relación con el nivel socioeconómico en adultos de la ciudad de Corrientes, Argentina. Rev Fac Nac Salud Pública. 2015;33(4).

6. Organizacion Mundial de la Salud. OMS | Salud bucodental; 2017. Available from: http://www.who.int/topics/ oral_health/es/. 
7. Henostroza G. Caries dental principios y procedimientos 17. Propdental. Los niveles de prevención en odontología. para el diagnostico. Lima; 2007.

8. González Rodríguez S, Pedroso Ramos L, Rivero Villalón M, Reyes Suárez VO. Epidemiología de la caClínicas Propdental. 2014 Apr;Available from: https://www.propdental.es/blog/odontologia/los-nivelesde-prevencion-en-odontologia.

ries dental en la población venezolana menor de 1918. UNICOC. Higiene Oral. Guía de práctica clínica en salud años. Medimay. 2014 Sep;20(2):208-218. Avai- oral. 2010;

lable from: http://revcmhabana.sld.cu/index.php/rcmh/19. Propdental. Consejos sencillos para mejorar la hiarticle/view/382/632.

9. Rada Silva HS, Quispe Ramos D. Enfermedades Periodontales Y Genetica. Revista de Actualización Clínica Investiga. 2013 05;31:1581 - 1585.20. Propdental. Cepillado dental mediante la técnica de Available from: http://www.revistasbolivianas. bass. Clínicas Propdental. 2013 Jan;Available from: https: org.bo/scielo.php?script=sci_arttext\&pid=S230437682013000400011\&nrm=iso.

10. Lorenzo S, Piccardo V, Alvarez F, Massa F, Alvarez R. Enfermedad Periodontal en la población joven y adulta uru- 22. Facultad de Odontología, Universidad Andrés Bellos. Maguaya del Interior del país: Relevamiento Nacional 20102011. Odontoestomatología. 2013 06;15:35 - 46. Availa- 23 ble from: http://www.scielo.edu.uy/scielo.php?script=sci_ arttext\&pid=S1688-93392013000200005\&nrm=iso.

11. Romero Castro N, Paredes Solís S, Legorreta Soberanis J, Reyes Fernández S, Flores Moreno M, Andersson N. Pre- 24. valencia de gingivitis y factores asociados en estudiantes de la Universidad Autónoma de Guerrero, México. Rev Cubana Estomatol. 2016;53(2):9-16.

12. Rojo Botello N, Flores Espinosa A, Arcos CastroII M. Prevalencia, severidad y extensión de periodontitis cróni- 25 . ca. Revista Odontológica Mexicana. 2011;15(1). Available from: http://www.revistas.unam.mx/index.php/rom/ article/view/23979.

13. Seguén Hernández J, Silva Guevara S, Ortiz Moncada C, Clavería Clark R, Font Jay MdlA. Evolución de la perio- 26. dontitis del adulto tratada con Apafill-G®. MEDISAN. 2013;17:971-980. Available from: https://www.redalyc. org/articulo.oa?id=368444993012.

14. Mafla AC, Muñoz GM, Barrera DA. Maloclusión y necesidad de tratamiento ortodóntico en adolescentes de Pasto, Colombia. Revista Facultad de Odontología Universidad de Antioquia. 2011;22(2).

15. Arroyo- Araya Yacsiry MHH. MALOCLUSIONES EN NIñOS DE 8 A 12 AñOS y LA PERCEPCIÓN DE LOS PADRES DE FAMILIA DE LA NECESIDAD DE UNA INTERCONSULTA CON EL ORTODONCISTA A TEMPRANA EDAD. Revista Científica Odontológica. 2012;

16. Bosch Robaina R, García Hoyos F, Rubio Alonso M. Conocimientos sobre salud bucodental y evaluación de higiene oral antes y después de una intervención educativa en niños de 9 y 10 años. AVANCES EN ODONTOESTOMATOLOGÍA. 2012;

Recibido: 19 de septiembre de 2018

Aceptado: 6 de diciembre de 2018 nual de higiene bucal. Sede Viña del Mar; 2015. Cueto-González N, Barreto D, Arce-Mena M, et al. Nivel de conocimiento sobre prevención de caries dental en universitarios. CES Odontologia. 2016;

. Reddy V, Bennadi D, Gaduputi S, Kshetrimayum N, Siluvai S, Reddy CVK. Oral health related knowledge, attitude, and practice among the pre-university students of Mysore city. J Int Soc Prev Community Dent. 2014 Sep;4(3):154-158.

. Pérez Oliva E. Conocimientos, actitudes y prácticas que posee la población universitaria de la UEES en relación a la Salud Bucal. Estudio Piloto realizado en el 2005. Rivera de Villacorta BL, editor; 2007. Available from: http://hdl.handle.net/10972/432.

. Park MK, Jin HJ, Lee MK. Investigation of knowledge and awareness of dental health in Chinese Students' Studying in Korea; 2014. p. 17-21.

Aceptado: 6 de diciembre de 2018

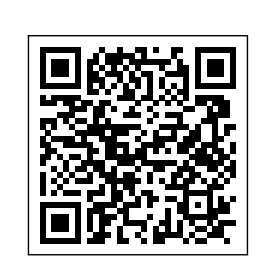

